\title{
Comparison of the Outcomes of Manual Small Incision Cataract Surgery (MSICS) and Phacoemulsification (PHACO) in Ghana
}

Samuel Kyei ${ }^{1}$, Ebenezer Zaabaar ${ }^{1}$, Frank Assiamah ${ }^{1}$, Michael Agyemang Kwarteng ${ }^{2}$, Kofi Asiedu ${ }^{3}$

1.Department of Optometry and Vision Science, School of Allied Health Sciences, College of Health and Allied Sciences, University of Cape Coast, Cape Coast, Ghana

2.Discipline of Optometry, University of KwaZulu-Natal, Durban, South Africa

3.Cosmopolitan Medical Center, Dzorwulu, Accra, Ghana

Correspondence to: Dr. Samuel Kyei; Email: skyei@ucc.edu.gh

Received: 15 Sept 2020; Revised: 9 Jan 2021; Accepted: 3 Feb 2021; Available online: 9 Mar 2021

\begin{abstract}
Background: The growing middle-class population of Ghana has seen more people being employed in visually demanding occupations and hence there is an increased desire for quality post-cataract surgical visual outcomes. This study aimed at comparing the outcomes of manual small incision cataract surgery (MSICS) and phacoemulsification (PHACO) among Ghanaians. Methods: This was a retrospective cross-sectional study in which records of patients who underwent MSCIS or phacoemulsification by the same surgeon were reviewed. Results: Medical records of 248 eyes were reviewed, out of which 132 underwent PHACO and 116 had MSICS. A significant number of the PHACO group had good (6/6-6/18) uncorrected visual acuity (UCVA) compared to the MSICS group at 1-2 weeks follow-up $(\mathrm{p}=0.003)$ and 4-6 weeks follow-up $(\mathrm{p}=0.002)$. MSICS resulted in a higher total astigmatic change compared to PHACO $(\mathrm{p}<0.001)$. The PHACO group
\end{abstract}

had a higher number of postoperative complications compared with the MSICS group ( $p<0.001)$. Postoperative borderline and poor uncorrected visual acuity were associated with age, total astigmatic change, and postoperative complications. Conclusion: The postoperative UCVA outcomes at 4-6 weeks' follow-up indicates that PHACO resulted in noticeably less spectacle dependency when compared to MSICS.

Keywords: Cataract, postoperative visual acuity, postoperative complications, total astigmatic change

Ann Afr Surg. 2021; 18(3): 143-149

DOI: http://dx.doi.org/10.4314/aas.v18i3.4

Conflicts of Interest: None

Funding: None

C 2021 Author. This work is licensed under the Creative Commons Attribution 4.0 International License.

\section{Introduction}

Despite efforts at tackling the menace of blindness due to cataract occurrence, it remains the leading cause of avoidable blindness worldwide, accounting for $51 \%$ of the global burden of blindness (1). In Ghana, cataracts are responsible for $54.8 \%$ of blindness nationwide (2). Cataract surgery has evolved from the earliest known method of couching to the recent approach of femtosecond laser-assisted cataract surgery (3).
Phacoemulsification (PHACO) is now the mainstay of cataract surgical extraction in the developed world and is the standard method of cataract extraction in regional centers in most developing countries (4), but manual small incision cataract surgery (MSICS) is the treatment modality that has gained popularity in Ghana.

However, the middle-class population of Ghana is growing $(5,6)$, and more people are now being 
employed in visually demanding occupations. There are now greater expectations concerning the quality of vision even among the retired. These visual demands associated with the changing economy of Ghana has ultimately led to patient dissatisfaction with late cataract extraction. Consequently, ophthalmology units in Ghana are gradually transitioning to PHACO, a technique that is ideally used for cataract removal at an early stage of its development.

Meanwhile, no survey has been conducted in Ghana to monitor the quality of this cataract surgical service or compare its outcomes to popular techniques that are already in place. This cataract surgery audit was, therefore, necessary to serve as an essential tool for monitoring the quality of cataract surgical services with PHACO and to determine whether the technique meets the high visual expectations of Ghanaians when compared with MSICS, to lay a basis for the presumed excellent price-quality ratio of PHACO (7) and to ensure that a high standard of cataract surgery is maintained to achieve targets related to the World Health Organization (WHO) recommended postoperative visual outcomes (8).

\section{Materials and methods}

This was a retrospective cross-sectional study conducted in ophthalmology units where the same surgeon operated on all patients. The study covered a period of 5 years, from January 2014 to December 2018. To control confounding variables except for refractive errors, records of all cataract surgeries performed on patients with any associated comorbidities like coexisting glaucoma, corneal pathology, uveitis, and other known pathologies (i.e., diabetes, hypertension, etc.) that could impair visual potential were excluded. Besides, surgical records involving traumatic cataract, combined procedures (cataract with penetrating keratoplasty, trabeculectomy, or strabismus surgery), and secondary intraocular lens (IOL) implantation were excluded from the study to control the influence of external variables. As all these were ensured, the visual defect was deemed to be attributable only to the cataract; hence, the same endpoint was expected in each technique.
Socio-demographic characteristics of patients, preoperative examination reports of patients, surgical details, and postoperative results were collated. Parameters studied included postoperative uncorrected visual acuity (UCVA) within $24 \mathrm{~h}$ of surgery, at 1-2 weeks' follow-up and 4-6 weeks' follow-up; total astigmatic change; postoperative best-corrected visual acuity (BCVA); intraoperative complications and number of postoperative complications.

Visual acuity was measured using Snellen's visual acuity chart and refractive errors were determined objectively using a handheld auto refractometer and subjectively by the maximum plus to maximum visual acuity method at $6 \mathrm{~m}$. Residual spherical refractive error was calculated by subtracting preoperative spherical power from postoperative spherical power; similarly, total astigmatic change was calculated by subtracting preoperative total cylinder power from postoperative total cylinder power. Visual acuities were categorized using the WHO guidelines on the outcome of cataract surgery: good (6/6-6/18), borderline $(6 / 24-6 / 60)$, and poor (worse than 6/60) (8).

Data analysis was done using the Statistical Package for Social Sciences (SPSS) for Windows, version 22.0 (Armonk, NY: IBM Corp.), and a p-value of $<0.05$ was considered statistically significant. Visual acuities and intraoperative complications between the two groups were compared using chi-square $(\chi 2)$ and Fisher's exact test. A non-parametric method (Mann-Whitney U test) was used to compare residual spherical refractive error, total astigmatic change, and the number of postoperative complications between the two techniques as data were not normally distributed. Multinomial logistic regression was also used to determine the potential risk factors for postoperative borderline and poor visual acuities.

The study was approved by the Institutional Review Board (IRB) of the University of Cape Coast (UCCIRB/CHAS/2019/195) and followed the tenets of the Declaration of Helsinki. As the medical records were reviewed retrospectively and identifying particulars of patients' details were concealed, patients' consents were not needed and were therefore waived by the IRB. All data and records generated throughout the course of the 
study were handled with strict confidentiality in alignment with the University of Cape Coast institutional policies.

\section{Results}

Records of 248 eyes operated on by the same surgeon fulfilled the inclusion criteria (Table 1). The mean age was 65.75 years [standard deviation $(\mathrm{SD})=9.85$ ]. A $\chi^{2}$ analysis of the outcomes of PHACO and MSICS in terms of UCVA and BCVA at different time intervals is as shown in Table 2.

Table 1. Gender distribution of sample population, preoperative BCVA and IOL position

\begin{tabular}{|c|c|c|c|}
\hline & $\begin{array}{l}\text { PHACO } \\
n(\%)\end{array}$ & $\begin{array}{l}\text { MSICS } \\
n(\%) \\
\end{array}$ & $\begin{array}{l}\text { TOTAL } \\
n(\%)\end{array}$ \\
\hline \multicolumn{4}{|l|}{ Gender } \\
\hline Males & $68(51.5)$ & $\begin{array}{l}50 \\
(43.1)\end{array}$ & $\begin{array}{l}118 \\
(47.6)\end{array}$ \\
\hline Females & $64(48.5)$ & $\begin{array}{l}66 \\
(56.9)\end{array}$ & $\begin{array}{l}130 \\
(52.4)\end{array}$ \\
\hline Total & $\begin{array}{l}132 \\
(100.0)\end{array}$ & $\begin{array}{l}116 \\
(100.0)\end{array}$ & $\begin{array}{l}248 \\
(100.0)\end{array}$ \\
\hline \multicolumn{4}{|l|}{ Preoperative BCVA } \\
\hline Good (6/6-6/18) & $92(69.7)$ & $3(2.6)$ & $95(38.3)$ \\
\hline $\begin{array}{l}\text { Borderline }(6 / 24- \\
6 / 60)\end{array}$ & $34(25.8)$ & $\begin{array}{l}15 \\
(12.9)\end{array}$ & $49(19.8)$ \\
\hline Poor $(<6 / 60)$ & $6(4.5)$ & $\begin{array}{l}98 \\
(84.5)\end{array}$ & $\begin{array}{l}104 \\
(41.9)\end{array}$ \\
\hline Total & $132(100.0)$ & $\begin{array}{l}116 \\
(100.0)\end{array}$ & $\begin{array}{l}248 \\
(100.0)\end{array}$ \\
\hline \multicolumn{4}{|l|}{ IOL position } \\
\hline$A C I O L$ & - & - & $14(5.65)$ \\
\hline PCIOL & - & - & $\begin{array}{l}134 \\
(94.35)\end{array}$ \\
\hline Total & & & $\begin{array}{l}248 \\
(100.00)\end{array}$ \\
\hline
\end{tabular}

ACIOL, anterior chamber intraocular lens; BCVA, best corrected visual acuity; IOL, intraocular lens; PCIOL, posterior chamber intraocular lens.

A Mann-Whitney U test was conducted to determine the difference in postoperative residual spherical refractive error and total astigmatic change between MSICS and PHACO. The results showed no significant difference in postoperative residual spherical refractive error $(\mathrm{p}=$
0.87). However, the analysis revealed that MSICS resulted in a significantly higher total astigmatic change compared to PHACO $(\mathrm{p}<0.001)$. Fisher's exact test showed no association between the rate of intraoperative complication and surgical technique (Table 3).

A Mann-Whitney $U$ test was used to investigate the difference in the number of postoperative complications between MSICS and PHACO. The analysis indicated that the number of postoperative complications recorded by each case was significantly greater in the PHACO group compared to the MSICS group ( $\mathrm{p}<0.001$ ). Several postoperative complications were recorded, the most often occurring being corneal edema followed by striate keratopathy (Figure 1). Table 4 shows the results of multinomial logistic regression analysis to determine potential risk factors for postoperative borderline and poor UCVA.

\section{Discussion}

Overall, there was an improvement in postoperative BCVA in both techniques. The outcomes of the current study were comparatively better than those of previous studies conducted in Ghana $(9,10)$. This could be attributed to the fact that the earlier studies did not include the outcomes of PHACO and did not exclude cases with preexisting ocular comorbidities. Besides, the previous studies did not provide records on postoperative best corrected visual acuity.

Similar to the results of the present study, Cook et al. (11) and Venkatesh et al. (12) observed that at postoperative day 1, UCVA was comparable between the PHACO group and the MSICS group. The difference observed in UCVA between the two techniques at 1 to 2 weeks follow-up in the current study might prove to be reasonable when compared with the study conducted by Khalaf et al. (13) who showed that PHACO produced a considerably good visual acuity at 1-2 weeks follow-up when compared with MSICS. UCVA and BCVA findings of the current study at 4-6 weeks follow-up corroborates the results of the study conducted by Gogate et al. (14) who reported a better UCVA outcome in the PHACO group compared to the MSICS group ( $p$ $=0.038$ ), and a comparable BCVA between the two groups. 
KYEI ET AL.

UCVA outcomes from both surgical techniques did not However, the PHACO group, as opposed to the MSICS measure up to the WHO benchmark of at least $80 \%$ of group, satisfied the WHO recommended standard of atpost-cataract patients attaining good UCVA (8).

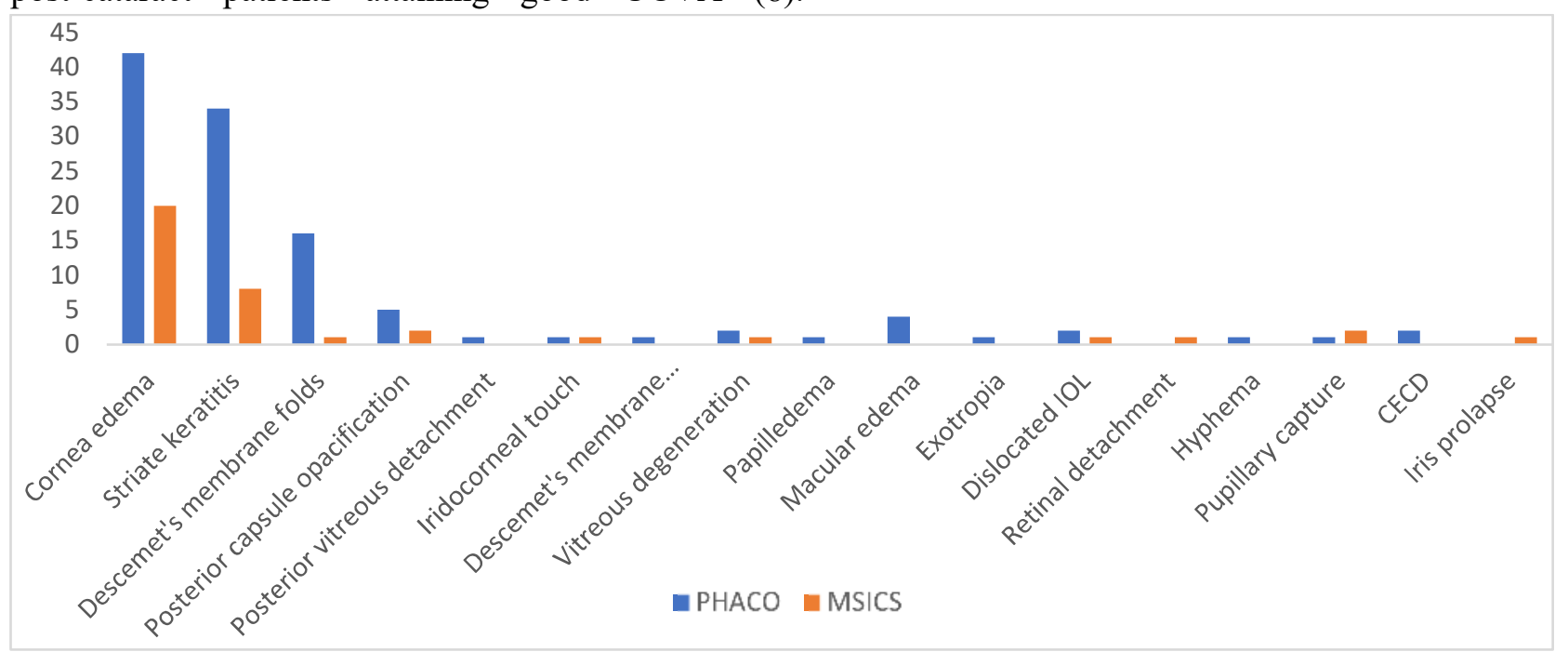

Figure 1. The total number of postoperative complications recorded in MSICS and PHACO. CECD, corneal endothelial cell density; IOL, intraocular lens; MSICS; manual small incision cataract surgery; PHACO, phacoemulsification.

Table 2. Association between surgical technique and postoperative UCVA and BCVA at different time intervals

\begin{tabular}{|c|c|c|c|c|c|}
\hline & PHACO $n(\%)$ & MSICS $n(\%)$ & Total $n(\%)$ & $\begin{array}{l}\text { Chi-square } \\
\left(\chi^{2}\right)\end{array}$ & $p$-value \\
\hline \multicolumn{6}{|c|}{ Uncorrected visual acuity at postoperative day 1} \\
\hline Good (6/6-6/18) & $48(36.4)$ & $33(28.4)$ & $81(32.7)$ & 2.773 & 0.253 \\
\hline Borderline (6/24-6/60) & $51(38.6)$ & $44(37.9)$ & $95(38.3)$ & & \\
\hline Poor $(<6 / 60)$ & $33(25.0)$ & $39(33.6)$ & $72(29.0)$ & & \\
\hline Total & $132(100.0)$ & $116(100.0)$ & $248(100.0)$ & & \\
\hline \multicolumn{6}{|c|}{ Uncorrected visual acuity at postoperative weeks $1-2$} \\
\hline Good (6/6-6/18) & $87(65.9)$ & $53(45.7)$ & $140(56.5)$ & 11.564 & 0.003 \\
\hline Borderline (6/24-6/60) & $33(25.0)$ & $52(44.8)$ & $85(34.3)$ & & \\
\hline Poor $(<6 / 60)$ & $12(9.1)$ & $11(9.5)$ & $23(9.3)$ & & \\
\hline Total & $132(100.0)$ & $116(100.0)$ & $248(100.0)$ & & \\
\hline \multicolumn{6}{|c|}{ Uncorrected visual acuity at postoperative weeks $4-6$} \\
\hline Good (6/6-6/18) & $96(72.7)$ & $59(50.9)$ & $155(62.5)$ & 12.606 & 0.002 \\
\hline Borderline (6/24-6/60) & $30(22.7)$ & $48(41.4)$ & $78(31.5)$ & & \\
\hline Poor $(<6 / 60)$ & $6(4.5)$ & $9(7.8)$ & $15(6.0)$ & & \\
\hline Total & $132(100.0)$ & $116(100.0)$ & $248(100.0)$ & & \\
\hline \multicolumn{2}{|c|}{ Best corrected visual acuity at postoperative weeks $4-6$} & \multicolumn{2}{|c|}{ Fisher's exact test } & & \\
\hline Good $(6 / 6-6 / 18)$ & $120(90.9)$ & $100(86.2)$ & $220(88.7)$ & 4.520 & 0.084 \\
\hline Borderline (6/24-6/60) & $12(9.1)$ & $12(10.3)$ & $24(9.7)$ & & \\
\hline Poor $(<6 / 60)$ & $0(0.0)$ & $4(3.4)$ & $4(1.6)$ & & \\
\hline Total & $132(100.0)$ & $116(100.0)$ & $248(100.0)$ & & \\
\hline
\end{tabular}


Outcomes Of Manual Small Incision Cataract SuRgery And Phacoemulsification

Table 3. Association between surgical technique and intraoperative complications

\begin{tabular}{lllll}
\hline COMPLICATIONS & PHACO $n(\%)$ & MSICS $n(\%)$ & Total $n(\%)$ & $p$-VALUE \\
\hline None & $126(95.5)$ & $114(98.3)$ & $240(96.8)$ & 0.19 \\
PCR with vitreous loss & $5(3.8)$ & $1(0.9)$ & $6(2.4)$ & \\
Zonular dialysis & $1(0.8)$ & $0(0.0)$ & $1(0.4)$ & \\
PCR without vitreous loss & $0.0(0)$ & $1(0.9)$ & $1(0.4)$ & \\
\hline Total & $132(100.0)$ & $116(100.0)$ & $248(100.0)$ & \\
\hline
\end{tabular}

PCR, posterior capsule rupture

Table 4. Factors associated with borderline and poor postoperative uncorrected visual acuities

\begin{tabular}{|c|c|c|c|c|}
\hline \multirow{2}{*}{$\begin{array}{l}\text { Postoperative } \\
\text { Week 4-6 UCVA variable }\end{array}$} & \multirow[b]{2}{*}{$P$-value } & \multirow[b]{2}{*}{ OR } & \multicolumn{2}{|c|}{$95 \%$ confidence interval } \\
\hline & & & Lower bound & Upper bound \\
\hline \multicolumn{5}{|l|}{ Borderline } \\
\hline NPOC & 0.005 & 1.651 & 1.161 & 2.348 \\
\hline Age & 0.224 & 1.019 & 0.988 & 1.051 \\
\hline TAC & 0.001 & 1.343 & 1.128 & 1.599 \\
\hline \multicolumn{5}{|l|}{ Gender } \\
\hline Male & 0.606 & 1.170 & 0.644 & 2.124 \\
\hline Female* & . & . & . & . \\
\hline \multicolumn{5}{|l|}{ IOC } \\
\hline Present & 0.410 & 1.966 & 0.394 & 9.815 \\
\hline Absent $^{*}$ & . & . & . & . \\
\hline \multicolumn{5}{|l|}{ IOL position } \\
\hline PCIOL & 0.822 & 1.160 & 0.317 & 4.238 \\
\hline$A C I O L^{*}$ & . & . & . & . \\
\hline \multicolumn{5}{|c|}{ Surgical technique } \\
\hline MSICS & 0.015 & 2.385 & 1.183 & 4.811 \\
\hline PHACO* & . & . & . & . \\
\hline \multicolumn{5}{|l|}{ Poor } \\
\hline NPOC & 0.008 & 2.158 & 1.219 & 3.821 \\
\hline Age & 0.037 & 1.068 & 1.004 & 1.136 \\
\hline TAC & 0.010 & 1.468 & 1.096 & 1.966 \\
\hline \multicolumn{5}{|l|}{ Gender } \\
\hline Male & 0.797 & 0.860 & 0.273 & 2.710 \\
\hline Female $^{*}$ & . & . & . & . \\
\hline \multicolumn{5}{|l|}{ IOC } \\
\hline Present & . & $1.140 \mathrm{E}-8$ & $1.140 \mathrm{E}-8$ & $1.140 \mathrm{E}-8$ \\
\hline Absent ${ }^{*}$ & . & . & . & . \\
\hline \multicolumn{5}{|l|}{ IOL position } \\
\hline PCIOL & 0.580 & 0.574 & 0.080 & 4.105 \\
\hline$A C I O L^{*}$ & . & . & . & . \\
\hline \multicolumn{5}{|c|}{ Surgical technique } \\
\hline $\begin{array}{l}\text { MSICS } \\
\text { PHACO }\end{array}$ & 0.361 & 1.970 & 0.460 & 8.438 \\
\hline
\end{tabular}

ACIOL, anterior chamber intraocular lens; IOC, intraoperative complications; IOL, intraocular lens; |NPOC, number of postoperative complications; PCIOL, posterior chamber intraocular lens; TAC, total astigmatic change. 
least $90 \%$ of post-cataract patients achieving good BCVA (8). Even though the postoperative UCVA of both surgical techniques did not match the WHO recommended standards (8), because many post-cataract patients in developing countries do not wear or cannot afford spectacles after surgery and thus rely on their UCVA to carry out their everyday functions $(9,15)$, it is worth stating that in the current study, UCVA at 4-6 weeks' follow-up suggested that PHACO reduced the dependency of many patients on distance spectacle correction or other interventions to correct ametropias compared to MSICS.

The significantly higher total astigmatic change in the MSICS group corroborates findings reported by some studies mentioned in the literature $(11,16,17)$. This difference in total astigmatic change between the two surgical techniques could be explained by the longer incision in MSICS. Other studies have, however, reported comparable surgically-induced astigmatism (SIA) between PHACO and MSICS (4, 12, 13). This inconsistency could be because previous studies compared surgically induced cornea astigmatism as opposed to the postoperative total astigmatic change compared in the current study.

Similar to the present study, other studies in the literature have reported higher proportions of posterior capsular rupture rates in the PHACO group versus the MSICS group $(18,19)$. The results, however, contrasts with the incidence in a tertiary eye care hospital in India, where a higher rate of posterior capsular rupture was recorded in the MSICS group (20). Notwithstanding, these rates of intraoperative complications were within the acceptable standard stated by the WHO (21).

In common with the current study, moderate-to-severe corneal edema was significantly higher in the PHACO group compared to the MSICS group $(p=0.001)$ at postoperative day 1 in a study conducted in Egypt (18). Similarly, Venkatesh et al. (12) showed that the MSICS group had less corneal edema than the PHACO group on the first postoperative day in cases with white cataracts. As seen in other studies, increasing age, residual ametropia (total astigmatic change) and postoperative complications were significant risk factors for borderline and poor outcomes (22-24). Despite excluding cases that had preexisting comorbidities, increasing age was found to be one of the risk factors of poor outcome. These older patients possibly had denser cataracts that precluded diagnoses of ocular comorbidities preoperatively.

The study was not immune to limitations. A major limitation is that the study is retrospective and nonrandomized and, therefore, does not provide the strongest and most direct epidemiologic evidence for causality. Besides, the relatively shorter period of follow-up possibly precluded the evaluation of longterm complications and the ultimate success rate of the two surgical techniques.

\section{Conclusion}

Early visual rehabilitation, less total astigmatic change, and a postoperative BCVA that meets the WHO acceptable standard were the major advantages of PHACO in this study. The UCVA at 4-6 weeks' followup suggests that PHACO resulted in a relatively less spectacle dependency compared to the MSICS group.

\section{Recommendations}

A prospective randomized controlled trial taking into consideration a larger and equally distributed sample size, with a relatively long follow-up period is recommended. To bridge the gap of visual demands in the changing economy of Ghana and to attain patient satisfaction especially among the middle- and upperclass population, consideration should be given to expanding more ophthalmology units to include the PHACO technique. However, MSICS should be maintained as an alternative for surgeons who may want to change procedures during surgery to attain better results and minimal complications in cases with very hard cataracts.

\section{Acknowledgment}

The authors are grateful to the surgeon who allowed us to audit his cases. 


\section{References}

1. Pascolini D, Mariotti, SP. Global estimates of visual impairment: 2010. Br J Ophthalmol. 2012; 96(5): 614618.

2. Wiafe B, Universal OE. Ghana blindness and visual impairment study. Accra: International Agency for the Prevention of Blindness, 2015.

3. Khurana AK. Comprehensive ophthalmology. 6th ed. New Delhi: The Health Sciences Publisher, 2015.

4. Ruit S, Tabin J, Chang D, et al. A prospective randomized clinical trial of phacoemulsification vs. manual sutureless small-incision extracapsular cataract surgery. Am J Ophthalmol. 2007; 143(1): 32-38.

5. Alada JJ, Umar MM, Alhassan MB, et al. Phacoemulsification: practice and barriers in Nigeria. Niger J Ophthalmol. 2017; 25(2): 101-114.

6. Ncube M, Lufumpa CL, Kayizzi-Mugerwa S. The middle of the pyramid: dynamics of the middle class in Africa. Market Brief. 2011;20.

7. Rochmah TN, Wulandari A, Dahlui $\mathrm{M}$, et al. Costeffectiveness analysis using disability-adjusted life years for cataract surgery. Int J Environ Res Public Health. 2020; 17: 1-9.

8. World Health Organization. WHO informal consultation on analysis of blindness prevention outcomes. Geneva: WHO/PBL/98/68, 1998.

9. Kobia-Acquah E, Pascal TM, Amedo A, et al. Visual outcome after cataract surgery at the Sunyani Regional Hospital, Ghana. ECOP. 2018; 9(4): 181-188.

10. Ilechie AA, Boadi-Kusi BS, Ndudiri OV, et al. Evaluation of post-operative visual outcomes of cataract surgery in Ghana. Int Health Res. 2012; 5(1): 35-42.

11. Cook C, Carrara H, Myer L. Phaco-emulsification versus manual small-incision cataract surgery in South Africa. S Afr Med J. 2012; 102(6): 537-40.

12. Venkatesh R, Tan SHC, Sengupta $S$, et al. Phacoemulsification versus manual small-incision cataract surgery for white cataract. J Cataract Refract Surg. 2010; 36: 1849-1854.

13. Khalaf M, Mohamed K, Anbar M, et al. Visual and astigmatic outcomes in manual small-incision cataract surgery versus phacoemulsification. Egypt $\mathrm{J}$ Cataract Refract Surg. 2016; 22: 37-41.

14. Gogate PM, Kulkarni SR, Krishnaiah S, et al. Safety and efficacy of phacoemulsification with manual smallincision cataract surgery by a randomized controlled clinical trial. Ophthalmology. 2005; 112(5): 869-74.

15. Gogate P. Clinical trial of manual small incision surgery and standard extracapsular surgery. Community Eye Health. 2003; 16(48): 54-55.

16. Harakuni U, Bubanale S, Smitha KS, et al. Comparison of surgically induced astigmatism with small incision cataract surgery and phacoemulsification. J Evol Med Dent Sci.2015; 4(71): 12354-12361.

17. Pai SG, Kamath SJ, Kedia V, et al. Cataract surgery in camp patients: a study on visual outcomes. Nepal J Ophthalmol. 2011;3(2): 159-164.
18. Enany AH. Phacoemulsification versus manual small incision cataract surgery in hard nuclear cataracts. Delta J Ophthalmol. 2018; 19(2): 92-98.

19. Haripriya A, Chang FD, Reena M, et al. Complication rates of phacoemulsification and manual small-incision cataract surgery at Aravind Eye Hospital. J Cataract Refract Surg. 2012; 38: 1360-1369.

20. Khanna RC, Kaza S, Shantha GPS, et al. Comparative outcomes of manual small incision cataract surgery and phacoemulsification performed by ophthalmology trainees in a tertiary eye care hospital in India: a retrospective cohort design. BMJ Open. 2012; 2(5).

21. Khanna RC, Rathi VM, Guizie E, et al. Factors associated with visual outcomes after cataract surgery: a crosssectional or retrospective study in Liberia. PLoS One. 2020; 15(5): e0233118.

22. Matta S, Park J, Palamaner Subash Shantha G, et al. Cataract surgery visual outcomes and associated risk factors in secondary level eye care centers of LV Prasad Eye Institute, India. PLoS One. 2016; 11(1): e0144853.

23. Lindfield R, Kuper H, Polack S, et al. Outcome of cataract surgery at one year in Kenya, the Philippines, and Bangladesh. Br J Ophthalmol. 2009; 93(7): 875-880.

24. Chak M, Wade A, Rahi JS. Long-term visual acuity and its predictors after surgery for congenital cataract: findings of the British congenital cataract study. Invest Ophthalmol Vis Sci. 2006; 47(10): 4262-4269. 